Check for updates

Cite this: RSC Adv., 2018, 8, 20499

\title{
Plasmon-driven surface catalytic reaction of 4- ethynylaniline in a liquid environment
}

\author{
Yu Liu, ${ }^{a}$ Caiqing $\mathrm{Ma},{ }^{a}$ Yanqiu Yang, ${ }^{a}$ Yuanchun Zhao, ${ }^{\text {a }}$ Shiwei Wu, ${ }^{\text {ac }}$ Jing Wang, ${ }^{a}$ \\ Peng Song (D) *b and Lixin Xia (D) *a
}

There is much evidence that surface plasmon photocatalytic reactions can occur on organic molecules on metal surfaces. In this paper, we focus on the photocatalytic reaction of 4-ethynylaniline (PEAN) on silver nanoparticles (Ag NPs) in a liquid environment by surface-enhanced Raman spectroscopy (SERS). Our experiments used SERS to characterize $p, p^{\prime}$-diynylazobenzene produced from PEAN via a selective catalytic coupling reaction on Ag NPs. This discovery not only achieved the expected results but also broadens the known plasmon-driven surface catalytic reaction system. In our work, we also regulated the photocatalytic coupling reaction conditions of PEAN on Ag NPs by laser power-dependent and time-dependent SERS spectra.

Received 18th April 2018 Accepted 29th May 2018

DOI: 10.1039/c8ra03326a

rsc.li/rsc-advances detection limit and good repeatability. ${ }^{9-12}$ More importantly, it also plays a major role in the qualitative and quantitative analyses of substances and molecular structures. At present, the main SERS mechanism generally recognized by the academic community includes physical enhancement mechanisms and chemical enhancement mechanisms. ${ }^{13}$ The former is a type of local electromagnetic field enhancement due to surface plasmon resonance (SPR), which is considered to be the most important contributor to achieving a greatly enhanced Raman signal. The latter mechanism, chemical enhancement, can be thought of as a chemical interaction between an analyte and a substrate, and the resulting charge-transfer (CT) inducedresonance Raman process. ${ }^{14}$ Electromagne-tic (EM) and chemical enhancement (CE) mechanisms are the two diffusely recognized mechanisms. The EM mechanism is induced by local surface plasmon polarisations, which can generally enhance the Raman spectra across a relatively large frequency region. In contrast, $\mathrm{CE}$ is induced by changes in the electronic structures of molecules adsorbed on the surface of the metal, which can selectively enhance certain Raman peaks. In general, the CE is interpreted by using the CT mechanism..$^{15}$

Comprehensive experimental studies have used SERS to examine 4-aminothiophenol adsorbed on the metal surface, and at the metal-molecule-metal connection system. ${ }^{16-20}$ For the first time, the enhancement mechanisms of the three markedly reinforced Raman peaks appearing in 1143, 1389, and $1430 \mathrm{~cm}^{-1}$ was explained as chemical mechanism by Osawa and co-workers in $1994 .^{21}$ After that, a large number of articles have been published, and these three markedly reinforced Raman peaks are commonly regarded as be empirical evidence of the SERS chemical mechanism..$^{22-24}$ However, such experimental SERS spectra of $p$-aminothiophenol (PATP) were not convincingly reproduced by simulations until 2009. In 2010, On the
${ }^{a}$ College of Chemistry, Liaoning University, Shenyang 110034, China. E-mail: lixinxia@lnu.edu.cn

${ }^{b}$ College of Physical, Liaoning University, Shenyang 110034, China. E-mail: songpeng@lnu.edu.cn

'Experimental Center of Shenyang Normal University, Shenyang 110034, China 
basis of density functional theory (DFT), Wu et al. speculated that the photocatalytic coupling reaction of PATP on the rough metal surface could produced $p, p^{\prime}$-dimercaptoazobenzene (DMAB); and the theoretical calculation normal Raman spectrum of DMAB is consistent with the experimental SERS spectrum of PATP. ${ }^{25}$ Subsequently, Sun and co-workers also fully studied the influence of the environment on the selective reaction of plasmon-driven and plasmon-exciton co-driven. ${ }^{26-28}$

Similarly, we selected 4-ethynylaniline to use as a probe molecule and $\mathrm{Ag}$ nanoparticles (NPs) as a substrate. Since the plasmon-driven chemical reactions in liquids are more efficient than in the atmosphere, ${ }^{29,30}$ we have investigated the selective plasma-driven oxidation of PEAN in a liquid environment using surface-enhanced Raman spectroscopy. We observed that $p, p^{\prime}$ diynylazobenzene (DYAB) was produced from 4-ethynylaniline (PEAN) via a surface photochemistry coupling reaction on silver nanoparticles. Interestingly, we observed the same reaction mechanism as for 4-aminothiophenol adsorbed on metal surfaces, and three obviously enhanced Raman peaks also appeared at $1138 \mathrm{~cm}^{-1}, 1390 \mathrm{~cm}^{-1}$ and $1432 \mathrm{~cm}^{-1}$, which were attributed to an $\mathrm{N}=\mathrm{N}$ vibration mode. We experimentally investigated the laser power-dependent and time-dependent SERS spectra of the plasmon-assisted surface-catalyzed dimerization of 4-ethynylaniline on Ag NPs.

\section{Experimental details}

Samples of PEAN and $\mathrm{AgNO}_{3}$ were purchased from Energy Chemical and used directly without further treatment or purification. Sodium citrate analytical reagent was purchased from J \& K Chemical.

The SERS-active Ag NPs were prepared by the sodium citrate reduction method as. First $0.018 \mathrm{~g}$ of silver nitrate particles $\left(\mathrm{AgNO}_{3}\right)$ were dissolved in a small amount of ultrapure water and diluted to $100 \mathrm{ml}$. Second transferred to a three-necked round bottom flask. Then it was heated to the state of micro-boiling under constant stirring in the oil bath. Finally, $2 \mathrm{ml}$ of $1 \%$ sodium citrate aqueous solution and a small amount of surfactant were quickly added. Subsequently, we could observe that the solution gradually changed from colorless and transparent to light yellow, then turned dark yellow, and finally formed a dark green Sol. The mixed solution was kept at a slightly boiling state for about $15 \mathrm{~min}$, then heating was stopped and the solution was allowed to cool to room temperature for use. Transmission electron microscopy (TEM) (Fig. 1b) image shows the particle size of the silver sol is about $40 \mathrm{~nm}$.

A solution with a concentration of $10^{-4}$ pairs of PEAN ethanol was prepared as follows. Some $1.2 \mathrm{mg}$ of PEAN was placed in a small beaker and dissolved in a small amount of anhydrous ethanol. Then it was transferred to a $100 \mathrm{ml}$ volumetric flask and enough ethanol was added to the mark to make $100 \mathrm{ml}$ of solution. The flask was placed in a cool, dark place for future use.

\section{Characterization}

The scanning electron microscope image was taken using a Japanese Hitachi Su8010 field emission scanning electron microscope.
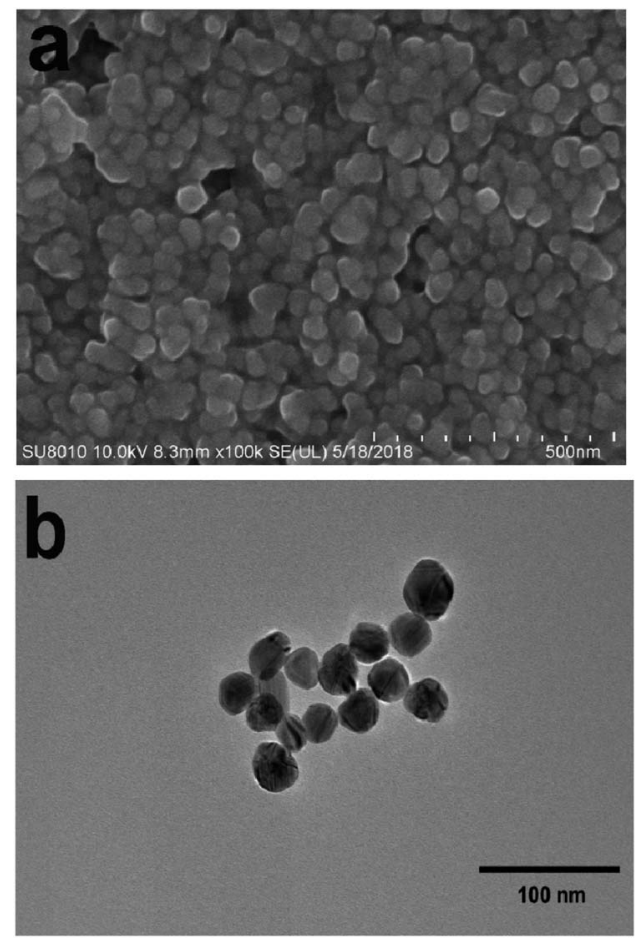

Fig. 1 (a) SEM image of Ag NPs; (b) TEM image of Ag NPs.

Transmission electron microscope images were taken using a Japanese JEM-2100 ultra-high resolution transmission electron microscope. UV-visible absorption spectra were collected using a PerkinElmer Lambda 35 UV/Visible spectrometer. The normal Raman spectroscopy was measured by using a capillary tube to draw a small amount of PEAN in ethanol solution. Similarly, SERS spectra experiments were performed by using a capillary to draw a mixture of PEAN and silver sol. We acquired laser intensitydependent and time-dependent SERS spectra from a Renishaw inVia Reflex confocal Raman system with a $50 \times(\mathrm{NA}=0.75)$ objective lens and a laser spot with a diameter of approximately 1 $\mu \mathrm{m}$. At a wavelength of $532 \mathrm{~nm}$, the laser's power was varied between $0.05 \mathrm{~mW}, 0.25 \mathrm{~mW}, 0.5 \mathrm{~mW}, 2.5 \mathrm{~mW}, 5.0 \mathrm{~mW}, 25 \mathrm{~mW}$, and $50 \mathrm{~mW}$, and exposure times of 10-120 s were used.

\section{Results and discussion}

We characterized the morphology and particle size of Ag NPs by scanning electron microscopy (SEM) and transmission electron microscopy (TEM). As shown in Fig. 1a, the SEM image of Ag NPs shows that their surface morphology is spherical. Fig. $1 \mathrm{~b}$ shows the TEM image of Ag NPs. It demonstrates that the particle size of $\mathrm{Ag}$ NPs is about $40 \mathrm{~nm}$.

In UV/Vis absorption spectra, metal nanoparticles give rise to a surface plasmon resonance absorption peak corresponding to the specific properties of the interband transition. The size and shape of the metal particles and the condensed state exert an influence on this peak. Moreover, the size distribution of metal particles affects the shape of the absorption lines. With a more uniform particle size distribution, the shape of the spectral line should be more symmetrical and narrower. From Fig. 2, it can 


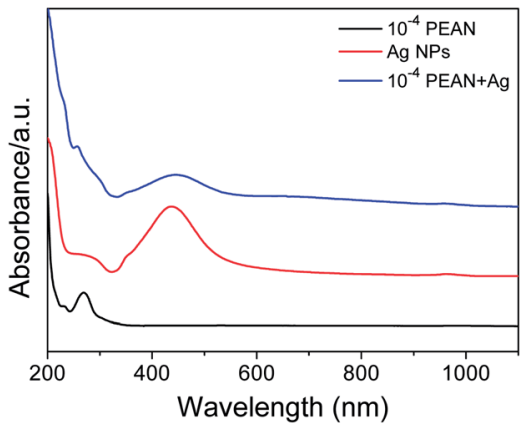

Fig. 2 UV/Vis absorption spectra of PEAN alcohol solution, Ag NPs and a 1 : 1 mixture of Ag NPs and PEAN, each in ethanol, from 200 to $1100 \mathrm{~nm}$.

clearly be seen that the Ag NPs (red line) gave rise to a strong absorption peak at around $430 \mathrm{~nm}$. The full-width at halfmaximum of this peak was narrow, indicating a uniform particle size distribution of the Ag NPs.

As can be seen from Fig. 2, the Ag NPs (red line) and the $1: 1$ mixture of Ag NPs and PEAN in ethanol (blue line) showed maxima at $430 \mathrm{~nm}$ and $450 \mathrm{~nm}$, respectively. This red-shift indicates that there is an interaction between the Ag NPs and the PEAN, causing the Ag NPs to agglomerate. Therefore, according to the UV absorption spectra, we chose to collect Raman spectra at a laser wavelength of $532 \mathrm{~nm}$.

Before conducting the experimental study of PEAN in a liquid environment, we first collected normal Raman spectra of PEAN powder as shown in Fig. 3. The strongest Raman peak near $2098 \mathrm{~cm}^{-1}$ is attributed to the stretching vibration mode of alkyne in PEAN. ${ }^{31}$ The Raman peak around $837 \mathrm{~cm}^{-1}$ was attributed to the benzene ring respiration, the $1179 \mathrm{~cm}^{-1}$ Raman peak was due to $\mathrm{C}-\mathrm{H}$ in-plane bending and the Raman peak of $1597 \mathrm{~cm}^{-1}$ was caused by C-C stretching modes. ${ }^{32}$ The Raman band at $1616 \mathrm{~cm}^{-1}$ was assigned to $-\mathrm{NH}_{2}$ scissor vibration. ${ }^{33}$

Fig. 4 shows the Raman spectrum of the $10^{-4} \mathrm{M}$ solution of PEAN in ethanol and the SERS spectrum of PEAN on Ag NPs. In the Raman spectrum, we did not observe a PEAN vibration peak. After adding Ag NPs, we observed some vibration bands under the same test conditions as shown in Fig. 4. In SERS spectrum, a strong band associated with the $\mathrm{C} \equiv \mathrm{C}$ stretching vibration at $1970 \mathrm{~cm}^{-1}$ was obviously red-shifted compared with that in the

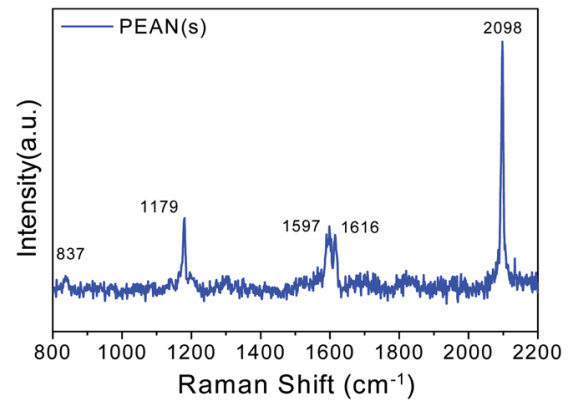

Fig. 3 Normal Raman spectroscopy of PEAN powder acquired at a laser wavelength of $532 \mathrm{~nm}$.

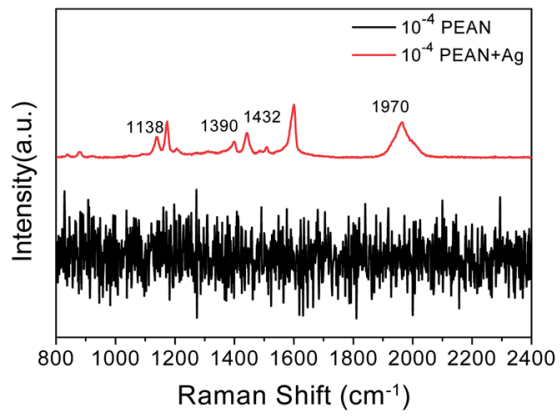

Fig. 4 Raman spectrum of $10^{-4} \mathrm{M}$ solution of PEAN in ethanol (black line); and SERS spectrum of PEAN on Ag NPs acquired with excitation at $532 \mathrm{~nm}$ (red line).

Raman spectrum, revealing powerful interactions between the alkyne carbon and the Ag NPs. Therefore, the SERS peak at $1970 \mathrm{~cm}^{-1}$ was regarded as a stable complex formed by the coordination of PEAN with Ag NPs surface. It is worth noting that new peaks appeared at $1138 \mathrm{~cm}^{-1}, 1390 \mathrm{~cm}^{-1}$, and $1432 \mathrm{~cm}^{-1}$. These peaks were not observed in the Raman spectrum of the PEAN powder (Fig. 3). We speculated that they were due to the formation of new products in photocatalytic reactions. These new Raman bands appeared at $1138 \mathrm{~cm}^{-1}$ due to $b(\mathrm{C}-\mathrm{H})$, and at $1390 \mathrm{~cm}^{-1}$ and $1432 \mathrm{~cm}^{-1}$ due to $v(\mathrm{~N}=\mathrm{N})$. More importantly, the shear vibration of $-\mathrm{NH}_{2}$ at $1616 \mathrm{~cm}^{-1}$ in the Raman spectrum of the PEAN powder disappeared. Based on the above results, we concluded that PEAN has a coupling reaction similar to PATP under the action of Ag NPs, in which 4ethynylaniline dimerizes to form DYAB.

Scheme 1 shows a schematic diagram of the reaction mechanism of PEAN dimerization into DYAB under the action of Ag NPs. According to previous reports, ${ }^{34,35}$ the role of surface plasmons in liquid and vacuum environments is different. This system shows that hot electrons excited by SPR can be transferred to the surrounding oxygen species, and that oxygen molecules are reduced to negative oxygen ions which, in turn, contribute to the oxidation of PEAN to DYAB ${ }^{36,37}$ In this process, PEAN loses the hydrogen proton and the simultaneous coupling reaction generates DYAB.

Next, we experimentally investigated the laser powerdependence and time-dependence of the plasmon-driven surface-catalyzed dimerization of PEAN on Ag NPs. Furthermore, we explored the best conditions for generating DYAB from PEAN.

Fig. 5 shows the SERS spectra of PEAN-Ag NPs acquired at an excitation of $532 \mathrm{~nm}$ at different power levels for durations of $10 \mathrm{~s}$. From the figure, we can observe splitting of the peak at $1970 \mathrm{~cm}^{-1}$ as the laser power increases. Weaver et al. described the "peak-splitting" phenomenon of $\mathrm{C} \equiv \mathrm{C}$ of acetylene on the surface of $\mathrm{Ag}$, and observed a band with a small split peak near the main peak $2020 \mathrm{~cm}^{-1}$, which did not move its Raman shift. The splitting of peaks is thought to be caused by low amounts of polymer formation. ${ }^{38}$ At the same time, we also observed that the three vibration mode peaks of $\mathrm{N}=\mathrm{N}$ are very obvious at a laser intensity of $0.5 \%$. With increasing laser intensity, the 


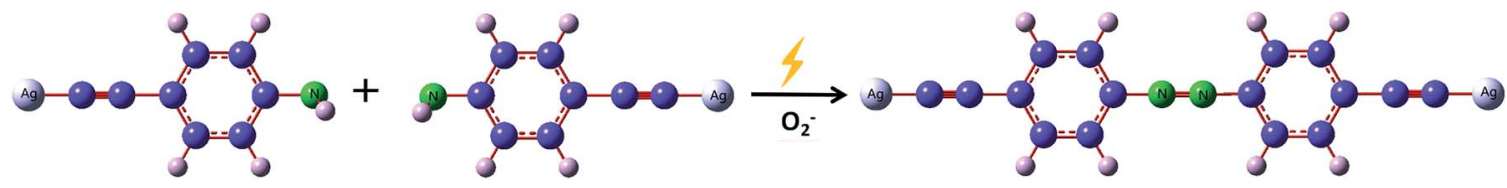

Scheme 1 Scheme for the proposed mechanism of Ag NPs induced dimerization of PEAN.

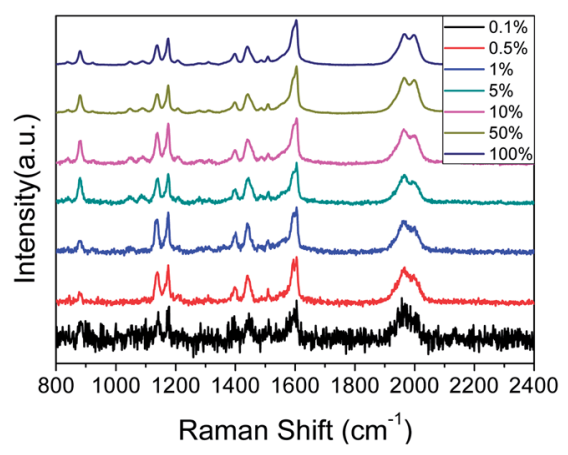

Fig. 5 Laser power-dependent surface-enhanced Raman spectra of PEAN-Ag NPs. Each spectrum was obtained with a 10 s exposure time.

three characteristic peaks of $\mathrm{N}=\mathrm{N}$ were gradually enhanced, and the $-\mathrm{NH}_{2}$ scissor vibration band gradually weakened. When the laser intensity reached $5 \%$, we clearly observed more product, and the $-\mathrm{NH}_{2}$ scissor vibration band at $1616 \mathrm{~cm}^{-1}$ disappeared completely. The above results show that PEAN is easily converted into DYAB under the action of plasmon resonance, which begins to occur at a laser intensity of $0.5 \%$. Because PEAN can be completely converted to DYAB when the laser intensity is $5 \%$, we chose to collect PEAN time-dependent SERS spectra at an excitation wavelength of $532 \mathrm{~nm}$ and a laser power of $2.5 \mathrm{~mW}$.
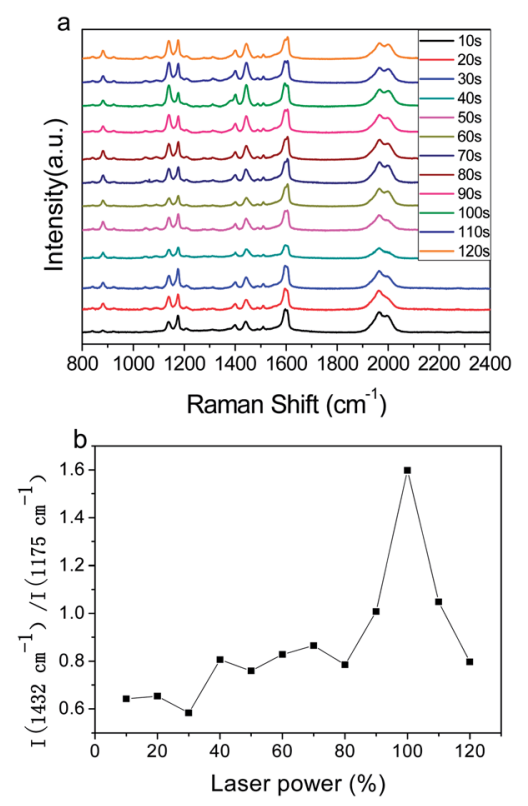

Fig. 6 (a) Time-dependent SERS spectra of PEAN-Ag NPs acquired at an excitation of $532 \mathrm{~nm}$ and power of $5 \%$ for durations of $10-120 \mathrm{~s}$. (b) Plot of $I_{\left(1432 \mathrm{~cm}^{-1}\right)} / I_{\left(1175 \mathrm{~cm}^{-1}\right)}$ according to laser power.
Fig. 6a shows the time-dependent SERS spectra of PEAN-Ag NPs acquired by excitation of $532 \mathrm{~nm}$ at a power of $5 \%$. The regularity of this time-dependent SERS spectrum is similar to that of the power-dependent SERS spectrum (Fig. 5). With increasing time, the intensity of the peaks at 1138, 1390 and $1432 \mathrm{~cm}^{-1}$ changed significantly. In addition, their Raman intensity gradually increased after the illumination time continued to increase. Fig. $6 \mathrm{~b}$ illustrates the relationship between the $1432: 1175 \mathrm{~cm}^{-1}$ intensity ratio and exposure time. From 10 to $100 \mathrm{~s}$, we can see that the relative intensity ratio increased and reached a maximum at $100 \mathrm{~s}$. During this period, there were two main processes. The first is the adsorption of PEAN molecules onto Ag NPs through the alkyne functional group, and the second is the formation of DYAB produced from PEAN on Ag NPs. The second step plays a leading role whereby several Ag NPs are assembled into small Ag NPs aggregates that can generate large electromagnetic fields. From 100 to 120 s, the process involves the aggregation of Ag NPs linked by the DYAB process, which shows a downward trend. This is because the agglomerated particles are too large, and only a few particles on the surface of the aggregate can sustain the enhancement of the SERS signal. ${ }^{39}$ Thence, based on the above experimental results and discussion, we believe that a laser power of $5 \%$ and an exposure time of $100 \mathrm{~s}$ are the optimal conditions for converting the product.

Fig. 7 shows the SERS spectra of PEAN mixed with different volumes of Ag NPs. It is obvious that the number of Ag NPs has a great influence on the Raman enhancement effect of PEAN. Under the same test conditions, the Raman enhancement effect decreases significantly with decreasing numbers of Ag NPs. From the experimental results, if the nano-silver ions are aggregated so that only a small amount of ions on the surface maintains the enhancement effect, the Raman signal enhancement of the molecules will be weakened. The experimental results demonstrate that the aggregation of Ag NPs connected by DYAB weakens the Raman signal.

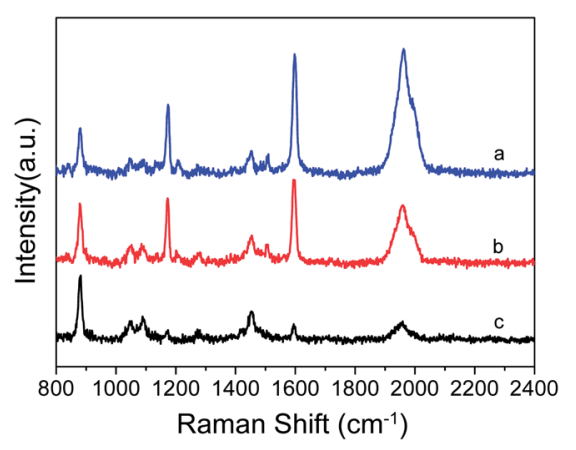

Fig. 7 SERS spectra of PEAN mixed with different volumes of Ag NPs; $V_{\text {PEAN }}: V_{\text {Ag NPs }}=$ (a) $2: 1$; (b) $3: 1$; (c) $4: 1$. 


\section{Conclusions}

In this work, we investigated the adsorption and reaction processes of PEAN on the surfaces of Ag NPs. We observed that PEAN and Ag NPs are connected by alkyne carbons, which DYAB produced from PEAN by selective catalytic coupling reactions on Ag NPs. This means that we can broaden the system of surface plasmon reaction. Not only does the thiol group interact with the metal surface, but also the alkyne group can bind well with the metal nanoparticles. From the laser-dependent and time-dependent SERS spectra of PEAN-Ag NPs, we have found a very interesting relationship in which the relative Raman intensity of the $b_{2}$ modes changed as the incident laser intensity or exposure time increased. In short, we have demonstrated that it is possible to study the reaction behavior of organic molecules under the action of metal nanoparticles and determine the optimal conditions for such reactions by surface-enhanced Raman spectroscopy.

\section{Conflicts of interest}

There are no conflicts to declare.

\section{Acknowledgements}

This work was supported by the National Natural Science Foundation of China (21671089 and 21271095), the Shenyang Natural Science Foundation of China (F16-103-4-00), the Scientific Research Fund of Liaoning Province (LT2017010, 20170540409), the Innovative Talent Support Program of Liaoning Province (Grant No. LR2017062), the Liaoning Provincial Department of Education Project (Grant No. LFW201710), the Natural Science Foundation of Liaoning Province (Grant No. 201602345)

\section{Notes and references}

1 J. Li, Z. W. Yang, B. Shao, J. Y. Liao, S. F. Lai, J. Q. Qiu, Z. G. Song and Y. Yang, J. Am. Ceram. Soc., 2015, 98, 15621566.

2 T. T. Tasi, T. W. Lin, L. D. Shao and H. H. Shen, RSC Adv., 2016, 6, 29453-29459.

3 Z. L. Zhang, D. Kinzel and V. Deckert, J. Phys. Chem. C, 2016, 120, 20978-20983.

4 X. P. Shen, T. J. Cui, D. Martin-Cano and F. J. Garcia-Vidal, Proc. Natl. Acad. Sci. U. S. A., 2013, 110, 40-45.

5 Y. F. Huang, M. Zhang, L. B. Zhao, J. M. Feng, D. Y. Wu, B. Ren and Z. Q. Tian, Angew. Chem., Int. Ed., 2014, 53, 2353-2357.

6 Q. Q. Ding, Y. Shi, M. D. Chen, H. Li, X. Z. Yang, Y. Q. Qu, W. J. Liang and M. T. Sun, Sci. Rep., 2016, 6, 32724.

7 T. W. Lin, T. T. Tasi, P. L. Chang and H. Y. Cheng, ACS Appl. Mater. Interfaces, 2016, 8, 8315-8322.

8 Q. Q. Ding, R. Li, M. D. Chen and M. T. Sun, Appl. Mater. Today, 2017, 9, 251-258.

9 H. C. Dai, Y. J. Sun, P. J. Ni, W. D. Lu, S. Jiang, Y. L. Wang, Z. Li and Z. Li, Sens. Actuators, B, 2017, 242, 260-268.
10 Y. F. Huang, H. P. Zhu, G. K. Liu, D. Y. Wu, B. Ren and Z. Q. Tian, J. Am. Chem. Soc., 2010, 132, 9244-9246.

11 S. S. Shen, B. Zhao, H. Y. Wang, Z. S. Li, G. Y. Qu, Z. N. Guo, T. L. Zhou, W. Song, X. Wang and W. D. Ruan, Colloids Surf., $A, 2014,443,467-472$.

12 M. Zhang, L. B. Zhao, W. L. Luo, R. Pang, C. Zong, J. Z. Zhou, B. Ren, Z. Q. Tian and D. Y. Wu, J. Phys. Chem. C, 2016, 120, 11956-11965.

13 Z. L. Zhang, S. X. Sheng, R. M. Wang and M. T. Sun, Anal. Chem., 2016, 88, 9328-9346.

14 J. L. Wang, I. D. Freitas, T. V. Alves, R. A. Ando, Z. B. Fang and P. H. Camargo, Chem.-Eur. J., 2017, 23, 7185-7190.

15 Y. Yin, P. Miao, Y. M. Zhang, J. C. Han, X. H. Zhang, Y. Gong, L. Gu, C. Y. Xu, T. Yao, P. Xu, Y. Wang, B. Song and S. Jin, Adv. Funct. Mater., 2017, 27, 1606694.

16 S. Duan, Y. J. Ai, W. Hu and Y. Luo, J. Phys. Chem. C, 2014, 118, 6893-6902.

17 P. Li, B. B. Ma, L. B. Yang and J. H. Liu, Chem. Commun., 2015, 51, 11394-11397.

18 G. K. Liu, J. Hu, P. C. Zheng, G. L. Shen, J. H. Jiang, R. Q. Yu, Y. Cui and B. Ren, J. Phys. Chem. C, 2008, 112, 6499-6508.

19 M. T. Sun and H. X. Xu, Small, 2012, 8, 2777-2786.

20 B. Kafle, M. Poveda and T. G. Habteyes, J. Phys. Chem. Lett., 2017, 8, 890-894.

21 M. Osawa, N. Matsuda, K. Yoshii and I. Uchida, J. Phys. Chem., 1994, 98, 12702-12707.

22 L. L. Kang, X. J. Han, J. Y. Chu, J. Xiong, X. He, H. L. Wang and P. Xu, ChemCatChem, 2015, 7, 1004-1010.

23 W. Ji, N. Spegazzini, Y. Kitahama, Y. J. Chen, B. Zhao and Y. Ozaki, J. Phys. Chem. Lett., 2012, 3, 3204-3209.

24 P. Xu, L. L. Kang, N. H. Mack, K. S. Schanze, X. J. Han and H. L. Wang, Sci. Rep., 2013, 3, 2997.

25 D. Y. Wu, X. M. Liu, Y. F. Huang, B. Ren, X. Xu and Z. Q. Tian, J. Phys. Chem. C, 2009, 113, 18212-18222.

26 L. Cui, X. Ren, X. Z. Yang, P. J. Wang, Y. Q. Qu, W. J. Liang and M. T. Sun, J. Raman Spectrosc., 2016, 47, 877-883.

27 W. H. Lin, X. Ren, L. Cui, H. Zong and M. T. Sun, Mater. Today, 2018, 11, 189-192.

28 W. H. Lin, Y. Q. Cao, P. J. Wang and M. T. Sun, Langmuir, 2017, 33, 12102-12107.

29 Q. Q. Ding, M. D. Chen, Y. Z. Li and M. T. Sun, Sci. Rep., 2015, 5, 10269.

30 E. Cao, X. Guo, L. Q. Zhang, Y. Shi, W. H. Lin, X. C. Liu, Y. R. Fang, L. Y. Zhou, Y. H. Sun and Y. Z. Song, Adv. Mater. Interfaces, 2017, 4, 1700869.

31 J. Wang, J. C. Dong, J. Yang, Y. Wang, C. J. Zhang, M. M. Xu, B. W. Mao, J. L. Yao, J. F. Li and Z. Q. Tian, Electrochem. Commun., 2017, 78, 16-20.

32 Y. J. Qi, Y. J. Hu, M. Xie, D. Xing and H. M. Gu, J. Raman Spectrosc., 2011, 42, 1287-1293.

33 L. X. Xia, C. Q. Ma, J. Wang, S. W. Wu, Y. Liu, Q. Zhang and P. Song, Chem. Commun., 2017, 53, 9582-9585.

34 Y. R. Fang, Z. L. Zhang and M. T. Sun, Rev. Sci. Instrum., 2016, 87, 033104.

35 Z. L. Zhang, P. Xu, X. Z. Yang, W. J. Liang and M. T. Sun, J. Photochem. Photobiol., C, 2016, 27, 100-112. 
36 J. L. Wang, R. A. Ando and P. H. Camargo, Angew. Chem., 2015, 127, 7013-7016.

37 L. Z. Papa, I. D. Freitas, R. S. Geonmonond, C. D. Aquino, J. C. Pieretti, S. H. Domingues, R. A. Ando and P. C. Camargo, J. Mater. Chem. A., 2017, 5, 11720-11729.
38 H. Feilchenfeld and M. J. Weaver, J. Phys. Chem., 1989, 93, 4276-4282.

39 Y. R. Fang, Y. Z. Li, H. X. Xu and M. T. Sun, Langmuir, 2010, 26, 7737-7746. 